\title{
Modeling the ionosphere-thermosphere response to a geomagnetic storm using physics-based magnetospheric energy input: OpenGGCM-CTIM results
}

\author{
Hyunju Kim Connor ${ }^{1,}$, Eftyhia Zesta ${ }^{1}$, Mariangel Fedrizzi ${ }^{2}$, Yong Shi ${ }^{3}$, Joachim Raeder ${ }^{4}$, Mihail V. Codrescu ${ }^{2}$, \\ and Tim J. Fuller-Rowell ${ }^{2}$ \\ 1 NASA Goddard Space Flight Center, Code 674, Building 21, Room 218, Greenbelt, MD 20771, USA \\ *Corresponding author: hyunju.k.connor@nasa.gov \\ 2 Space Weather Prediction Center, National Oceanic and Atmospheric Administration, Boulder, Colorado 80305, USA \\ 3 Department of Atmospheric and Oceanic Sciences, University of California, Los Angeles, California 90095, USA \\ 4 Space Science Center, University of New Hampshire, Durham, NH 03824, USA
}

Received 16 June 2015 / Accepted 9 May 2016

\begin{abstract}
The magnetosphere is a major source of energy for the Earth's ionosphere and thermosphere (IT) system. Current IT models drive the upper atmosphere using empirically calculated magnetospheric energy input. Thus, they do not sufficiently capture the stormtime dynamics, particularly at high latitudes. To improve the prediction capability of IT models, a physics-based magnetospheric input is necessary. Here, we use the Open Global General Circulation Model (OpenGGCM) coupled with the Coupled Thermosphere Ionosphere Model (CTIM). OpenGGCM calculates a three-dimensional global magnetosphere and a two-dimensional high-latitude ionosphere by solving resistive magnetohydrodynamic (MHD) equations with solar wind input. CTIM calculates a global thermosphere and a high-latitude ionosphere in three dimensions using realistic magnetospheric inputs from the OpenGGCM. We investigate whether the coupled model improves the storm-time IT responses by simulating a geomagnetic storm that is preceded by a strong solar wind pressure front on August 24, 2005. We compare the OpenGGCM-CTIM results with low-earth-orbit satellite observations and with the model results of Coupled Thermosphere-Ionosphere-Plasmasphere electrodynamics (CTIPe). CTIPe is an up-to-date version of CTIM that incorporates more IT dynamics such as a low-latitude ionosphere and a plasmasphere, but uses empirical magnetospheric input. OpenGGCM-CTIM reproduces localized neutral density peaks at $\sim 400 \mathrm{~km}$ altitude in the high-latitude dayside regions in agreement with in situ observations during the pressure shock and the early phase of the storm. Although CTIPe is in some sense a much superior model than CTIM, it misses these localized enhancements. Unlike the CTIPe empirical input models, OpenGGCM-CTIM more faithfully produces localized increases of both auroral precipitation and ionospheric electric fields near the high-latitude dayside region after the pressure shock and after the storm onset, which in turn effectively heats the thermosphere and causes the neutral density increase at $400 \mathrm{~km}$ altitude.
\end{abstract}

Key words. Space Weather - Magnetosphere - Ionosphere - Thermosphere - Modeling

\section{Introduction}

The magnetosphere is one of the major energy sources that drive the Earth's upper atmosphere. During storm times, the magnetosphere deposits significant energy into the ionosphere and thermosphere (IT) in the form of auroral particle precipitation and Poynting flux. The subsequent high-latitude ionization, ionospheric electric field variations, Joule heating, and ion-drag forcing alter the global ionosphere-thermosphere dynamics. For a summary of storm-time IT responses, see Richmond \& Lu (2000) and Fuller-Rowell et al. (2007).

The development of physics-based IT models and model validation with low-Earth orbit (LEO) satellite observations have improved our ability to predict the global IT system. Most models drive the IT system using empirically, statistically, or analytically calculated magnetospheric input. The Weimer (Weimer 2005) and Heelis models (Heelis et al. 1982) are often used to calculate ionospheric electric fields. The TIROSNOAA statistical aurora maps (Fuller-Rowell \& Evans 1987) and a simple analytic aurora model (Roble \& Ridley 1987) are used for the aurora precipitation input. Since these input models average over decades of observations or provide simple analytic solutions, they can be fast input solvers for any type of activity level or location, but cannot sufficiently capture the spatiotemporal dynamics of magnetospheric energy sources during geomagnetic active times, thus limiting the predictive capability of IT models when it is most needed.

More realistic input sources are available. The Assimilative Mapping of Ionospheric Electrodynamics (AMIE) technique (Richmond \& Kamide 1988), for example, provides ionospheric electrodynamic variables by assimilating the observations from radars, ground magnetometers, and LEO satellites. Crowley et al. (2010) ingested the AMIE electric fields into the Thermosphere Ionosphere Mesosphere Electrodynamics General Circulation Model (TIMEGCM), and reproduced the high-latitude thermospheric density enhancement observed by the Challenging Minisatellite Payload (CHAMP) spacecraft after a sudden increase of IMF $B_{y}$, while Schlegel et al. (2005) failed to simulate a similar density enhancement by using the same IT model but with empirical magnetospheric 
input. Still, the accuracy of AMIE results is limited as well because it strongly depends on data coverage and it is poor where data coverage is poor (Knipp \& Emery 1997).

The global magnetosphere-ionosphere MHD models provide an alternative way to calculate the magnetosphere sources. MHD models calculate ionospheric electric fields by assuming a closure of field-aligned currents in the ionosphere. They also calculate auroral precipitation by ingesting MHD plasma quantities to the electron precipitation equations described by Knight (1973), Lyons et al. (1979), and Kennel \& Petschek (1966) for example. There are few magnetosphere-ionosphere-thermosphere coupled models. The coupled magnetosphere-ionosphere-thermosphere (CMIT) model links the Lyon-Fedder-Mobarry (LFM) global magnetosphere MHD model to the Thermosphere Ionosphere Electrodynamics General Circulation Model (TIEGCM) model (Wang et al. 2008). Using the magnetospheric input from the LFM MHD model, CMIT can produce penetration of high-latitude electric fields near the magnetic equator (Wang et al. 2008), storm-time positive and negative responses of total electron content (Lei et al. 2008; Wang et al. 2010), and realistic timing of IT responses to IMF variations (Wang et al. 2004).

The OpenGGCM-CTIM is another coupled magnetosphere-ionosphere-thermosphere model developed in the late 1990s by linking OpenGGCM and CTIM. The coupled model produces reasonable ionospheric responses such as ionospheric conductance (Raeder et al. 2001b), Joule heating (Li et al. 2011), proton aurora precipitation (Gilson et al. 2012), and cross polar cap potentials (Raeder \& Lu 2005; Connor et al. 2014) during various solar wind and IMF conditions. However, the previous studies have focused on the improvement of the OpenGGCM magnetospheric response that resulted from the coupling with CTIM, while the impact of the coupling was on. The coupling impact on the IT responses has not been explored.

Motivated by the CMIT studies (Wang et al. 2004, 2008, 2010; Lei et al. 2008) and the AMIE-TIMEGCM studies (Crowley et al. 2010), this paper investigates the thermospheric responses of OpenGGCM-CTIM by simulating the geomagnetic storm on August 24, 2005. We compare the OpenGGCM-CTIM results with LEO satellite observations and the model results of CTIPe, the up-to-date model of CTIM that uses empirical magnetospheric input. The model-to-data comparison in this paper is conducted in a qualitative way to test if models follow general trends of observations. This paper highlights the importance of including physics-based magnetospheric input parameters in the IT simulation.

\section{Models}

\subsection{OpenGGCM-CTIM}

The OpenGGCM-CTIM is a two-way coupled global magnetosphere-ionosphere-thermosphere (MIT) model composed of the OpenGGCM and CTIM. OpenGGCM simulates the three-dimensional global magnetosphere and includes a twodimensional height-integrated electrodynamic high-latitude ionosphere submodel. The outer magnetosphere part of the model solves the resistive MHD equations with solar wind and IMF input. CTIM simulates the three-dimensional global thermosphere and the three-dimensional high-latitude ionosphere by solving neutral and ion fluid equations with solar radiation, tidal modes, and magnetospheric energy input. For a low-latitude ionosphere below $23^{\circ}$ geographic latitude, CTIM uses a empirical model of Chiu (1975).

OpenGGCM provides auroral precipitation and ionospheric electric fields to CTIM. It calculates two types of electron precipitation, diffuse and discrete aurora, using plasma parameters and field-aligned currents near the inner magnetosphere boundary located at 3.5 Re from the Earth's center. Diffuse aurora precipitation occurs when the plasmasheet electrons enter the pitch-angle loss cone. OpenGGCM assumes a complete pitch-angle scattering of electrons at the inner magnetosphere boundary, calculating the mean energy $E_{0}$ and energy flux $F_{\mathrm{E}}$ of diffuse aurora (Kennel \& Petschek 1966):

$$
\begin{gathered}
E_{0}=k T_{\mathrm{e}}, \\
F_{\mathrm{E}}=\alpha n_{\mathrm{e}} k T_{\mathrm{e}}\left(k T_{\mathrm{e}} / 2 \pi m_{\mathrm{e}}\right)^{1 / 2},
\end{gathered}
$$

where $k$ is the Boltzmann constant, $T_{\mathrm{e}}, n_{\mathrm{e}}$, and $m_{\mathrm{e}}$ are electron temperature, density, and mass, and $\alpha$ is a fudge factor to adjust energy flux. Since OpenGGCM provides only proton parameters, it assumes $T_{\mathrm{e}}$ and $n_{\mathrm{e}}$ have the same temperature and density of protons. Discrete aurora precipitation occurs when electrons are accelerated by parallel potential drops along magnetic field lines. The energy $E_{0}$ and energy flux $F_{\mathrm{E}}$ (Knight 1973; Lyons et al. 1979) are:

$$
\begin{gathered}
E_{0}=k T_{\mathrm{e}}+e \Delta \Phi_{\|}, \\
F_{\mathrm{E}}=\beta \Delta \Phi_{\|}\left|J_{\|}\right|, \\
\Delta \Phi_{\|}=e^{2} n_{\mathrm{e}} /\left(2 \pi m_{\mathrm{e}} k T_{\mathrm{e}}\right)^{1 / 2} \max \left(0,-J_{\|}\right),
\end{gathered}
$$

where $J_{\|}$is field-aligned current densities, $\Delta \Phi_{\|}$is parallel potential drops, and $\beta$ is a fudge factor to adjust energy flux. OpenGGCM produces the discrete aurora precipitation only in the regions of upward field-aligned currents.

OpenGGCM calculates ionospheric electric fields by employing current continuity in the ionosphere, i.e., using the well-known ionospheric electric field equation (Vasyliunas 1970; Kelley 1989):

$$
\nabla \cdot(\underline{\boldsymbol{\Sigma}} \cdot \boldsymbol{E})=-J_{\|} \sin I
$$

where $\boldsymbol{\Sigma}$ is the ionospheric conductance tensor, $\boldsymbol{E}$ is the ionospheric electric field, and $I$ is the inclination of dipole field in the ionosphere. OpenGGCM calculates $J_{\|}$by mapping the field-aligned currents from the inner magnetosphere boundary to the ionosphere along dipole field lines. CTIM calculates more realistic ionospheric conductance $(\underline{\boldsymbol{\Sigma}})$ than the empirical conductance model of Robinson et al. (1987) or a uniform conductance model that were employed in OpenGGCM prior to the coupling with CTIM (see Raeder et al. 2001b). The so-obtained ionospheric electric fields are not only used to drive the ionosphere and thermosphere, but are also fed back to the inner magnetosphere boundary by mapping the electric fields from the ionosphere to the boundary along dipole magnetic field lines, thus closing the magnetosphere-ionosphere convection cycle. More details are provided in Raeder et al. (2001a, 2001b, 2008) and Raeder (2003). 


\subsection{Coupled Thermosphere-Ionosphere-Plasmasphere electrodynamics (CTIPe)}

The CTIPe model is an advanced version of CTIM obtained by adding mid- and low-latitude ionosphere, plasmasphere, and global dynamo electric field calculations. CTIPe uses statistical and empirical models for the magnetospheric energy input. The TIROS-NOAA satellite measurements (Fuller-Rowell \& Evans 1987) provide seven aurora patterns that are keyed to the hemispheric power index (HPI). Since the HPI activity level ranges from 1 to 10 , the weakest aurora map is shared when HPI is less than 4 and the strongest aurora map is shared when HPI is greater than 8 . The Weimer ionosphere electrodynamics model (Weimer 2005) provides empirical patterns of electric potential using solar wind and IMF conditions as input. Because these inputs are based on long-term averages, it is difficult to catch particular temporal and spatial variation of magnetospheric energy input during a geomagnetic storm, thus limiting the predictability of CTIPe. For more details on CTIPe, see Fuller-Rowell et al. (1996), Millward et al. (1996, 2001), and Codrescu et al. (2012).

The best way to understand the role of a physics-based magnetospheric input would be to compare the OpenGGCMCTIM results with the CTIM results. However, the stand-alone CTIM is no longer available, so we use CTIPe as an alternative for the model-to-model comparison. Because CTIPe and CTIM use the same physics models to calculate global thermosphere and high-latitude ionosphere, differences between the two model results are minimal especially in the high-latitude region if the same magnetospheric energy input is used (T. J. Fuller-Rowell, private communication, 2013).

\section{Data}

For model-data comparison purpose, we use thermospheric mass density deduced from the Challenging Minisatellite Payload (CHAMP) and the Gravity Recovery and Climate Experiment (GRACE) satellites. CHAMP was launched in July 2000 into a near-polar orbit at $425 \mathrm{~km}$ altitude with $87.25^{\circ}$ inclination and 93 min orbital period. During its 10 years of operations the CHAMP altitude slowly degraded to $\sim 311 \mathrm{~km}$ and the mission ended in September 2010 with re-entry. The GRACE twin satellites were launched in March 2002 into a near-circular polar orbit at $\sim 500 \mathrm{~km}$ altitude with $89.5^{\circ}$ inclination, $95 \mathrm{~min}$ orbital period, and $\sim 220 \mathrm{~km}$ spacecraft separation. Due to atmospheric drag, the GRACE orbit has been gradually decaying to $\sim 387 \mathrm{~km}$ altitude as of May 13, 2015 based on the GRACE website at http://www.csr.utexas.edu/ grace/.

We also use total electric field, auroral energy flux, and Poynting flux calculated from the Defense Meteorological Satellite Program (DMSP) spacecraft observations. The DMSP satellites operate in a near-polar, sun-synchronous orbit at $\sim 830 \mathrm{~km}$ altitude with $\sim 101 \mathrm{~min}$ orbital period. The two DMSP instruments, Special Sensor Magnetometer (SSM) and the Special Sensor-Ion and Electron Scintillation (SSIES), provide measurements for the Poynting flux calculation. The Poynting flux $(\boldsymbol{S})$ is derived in a spacecraft coordinate system using $\boldsymbol{S}=\boldsymbol{E} \times \boldsymbol{\delta} \boldsymbol{B} / \mu_{0}$. The electric field $(\boldsymbol{E})$ and magnetic perturbation $(\boldsymbol{\delta} \boldsymbol{B})$ are determined from $\boldsymbol{E}=-\boldsymbol{V} \times \boldsymbol{B}_{\mathrm{IGRF}}$ and $\boldsymbol{\delta} \boldsymbol{B}=\boldsymbol{B}_{\mathrm{SSM}}-\boldsymbol{B}_{\mathrm{IGRF}}$, where $\boldsymbol{V}$ is the plasma drift velocity from the SSIES instrument, $\boldsymbol{B}_{\mathrm{SSM}}$ is the magnetic field vector from the SSM instrument, and $\boldsymbol{B}_{\mathrm{IGRF}}$ is International Geomagnetic
Reference Field (IGRF) magnetic fields obtained at the spacecraft location. This paper uses vertical Poynting flux, $\boldsymbol{S}_{\|}=\left(\boldsymbol{E}_{x} \boldsymbol{\delta} \boldsymbol{B}_{y}-\boldsymbol{E}_{y} \boldsymbol{\delta} \boldsymbol{B}_{x}\right) / \mu_{0}$, where $x$ and $y$ refer to the components along and across the satellite track.

\section{Results and discussion}

We run the OpenGGCM-CTIM and CTIPe models to reproduce the thermospheric mass density observed by CHAMP and GRACE during the geomagnetic storm on August 24, 2005. Figures 1a-1c show solar wind and IMF conditions in geocentric solar ecliptic coordinates obtained from the OMNI website at http://omniweb.gsfc.nasa.gov/. Solar wind (SW) and IMF conditions are relatively constant until 6:10 UT when the solar wind dynamic pressure $\left(P_{\mathrm{sw}}\right)$ suddenly increases while the IMF turns strongly duskward and weakly northward. The $P_{\mathrm{sw}}$ sharply drops at 9:10 UT while the IMF $B_{z}$ turns southward and IMF $B_{y}$ strongly duskward which mark the onset of storm conditions. The SYM/H index, not shown in this paper, starts decreasing at this time and reaches $\sim-180 \mathrm{nT}$ near 11:50 UT. The two vertical black lines in Figure 1 indicate the $P_{\text {sw }}$ shock arrival and the storm onset.

Figures 1d-1g show, for the full $24 \mathrm{hrs}$ on August 24, 2005, model-data comparison of CHAMP thermospheric mass density, model-data comparison of GRACE thermospheric mass density, magnetic latitude (MLAT) of CHAMP and GRACE, and magnetic local time (MLT) of CHAMP and GRACE, respectively. The red, blue, and green lines in Figures $1 \mathrm{~d}$ and 1e represent observations from CHAMP/GRACE, CTIPe results, and OpenGGCM-CTIM results. The model results of neutral densities are obtained along the respective spacecraft trajectories. The blue and green lines in Figures $1 \mathrm{f}$ and $1 \mathrm{~g}$ represent orbit information of CHAMP and GRACE, respectively. Figure 2 also presents the same model-data comparison of thermospheric mass densities, but focusing on more details during the 5:00-13:00 UT period. Figures 2a-2e display SW/ IMF conditions, model-data comparison of CHAMP neutral density, CHAMP orbit information, model-data comparison of GRACE neutral density, and GRACE orbit information, respectively. The labels of colored lines appear on the upper left corner of each panel. All the neutral density data presented in this paper are normalized to $400 \mathrm{~km}$ altitude for easy comparison.

During the storm CHAMP's orbit ascends on the dayside at $\sim 12$ MLT and descends on the nightside at $\sim 0$ MLT, while GRACE ascends near 15 MLT and descends near 3 MLT (see Fig. 3d for the polar view of CHAMP and GRACE orbits). Before the shock impact at 6:10 UT, both CHAMP and GRACE show typical sinusoidal day-night variations in Figures 1 and 2. The sudden enhancement of solar wind pressure (Y. Shi, private communication, 2012) and the increase of IMF $B_{y}$ (Knipp et al. 2011) disturb magnetospheric magnetic fields, producing Poynting flux into the high-latitude ionosphere and thus heating the nearby thermosphere. During the strong $P_{\mathrm{sw}}$ period (6:10-9:10 UT), both spacecraft observe increased thermospheric mass densities at the high-latitude dayside regions compared to the densities at the same regions before the $P_{\mathrm{sw}}$ impact. To guide the eye, the magenta markers in Figures 1 and 2 point to the density peaks during the strong $P_{\text {sw }}$ period.

The magnetic storm starts around 9:10 UT when the IMF $B_{z}$ abruptly decreases. The thermospheric density significantly enhances at high latitudes during the initial stage of the storm (9:10-12:10 UT), showing much stronger density peaks 
MODEL-DATA Comparison on 2005 Aug 24

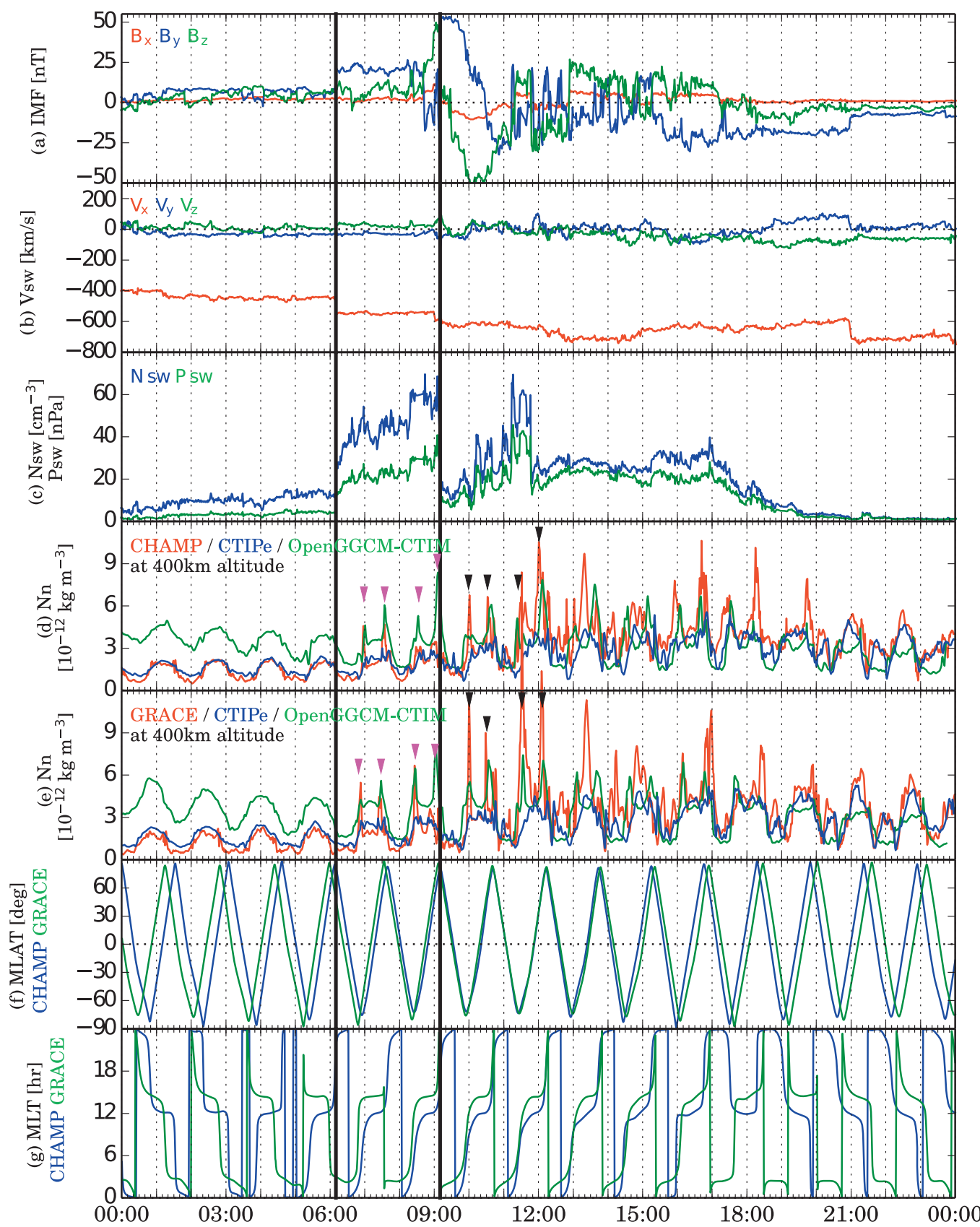

Fig. 1. Model-data comparison during a whole storm day on August 24, 2005. (a) IMF, (b) solar wind velocity, (c) solar wind number density (blue) and dynamic pressure (green), (d) CHAMP thermospheric mass densities (red) compared with the model results from CTIPe (blue) and OpenGGCM-CTIM (green), (e) GRACE thermospheric mass densities (red) compared with the model results from CTIPe (blue) and OpenGGCM-CTIM (green), (f) magnetic latitude of CHAMP (blue) and GRACE (green), and (g) magnetic local time of CHAMP (blue) and GRACE (green). The magenta and black markers point to the density peaks during the strong $P_{\text {sw }}$ period and the storm time, respectively.

compared to the ones during 6:10-9:10 UT. The black markers in Figures 1 and 2 point to the storm-time density peaks. At the later stage of the storm, the high-latitude thermospheric heating initiates a new global circulation and redistributes the neutral and plasma heating and disturbances over the globe. The enhanced thermospheric density moves from both poles to lower latitudes (Richmond et al. 2000), creating density peaks near the equator in both the CHAMP and GRACE observations (for example, see the density peak at $\sim 13: 20$ UT in Figs. 1d and 1e).
We compare the satellite observations with the CTIPe and OpenGGCM-CTIM results in Figures 1d and 1e. While the observations demonstrate intense localized heating of the thermosphere at polar latitudes immediately after the SW driver (shock or storm), such response is absent from the CTIPe model. CTIPe captures the thermospheric density really well during the quiet time before 6:10 UT, but does not produce the localized, high-latitude density peaks observed after 6:10 UT. In contrast, the coupled OpenGGCM-CTIM model drives the IT system using more realistic magnetospheric 
MODEL - DATA Comparison on 2005 Aug 24

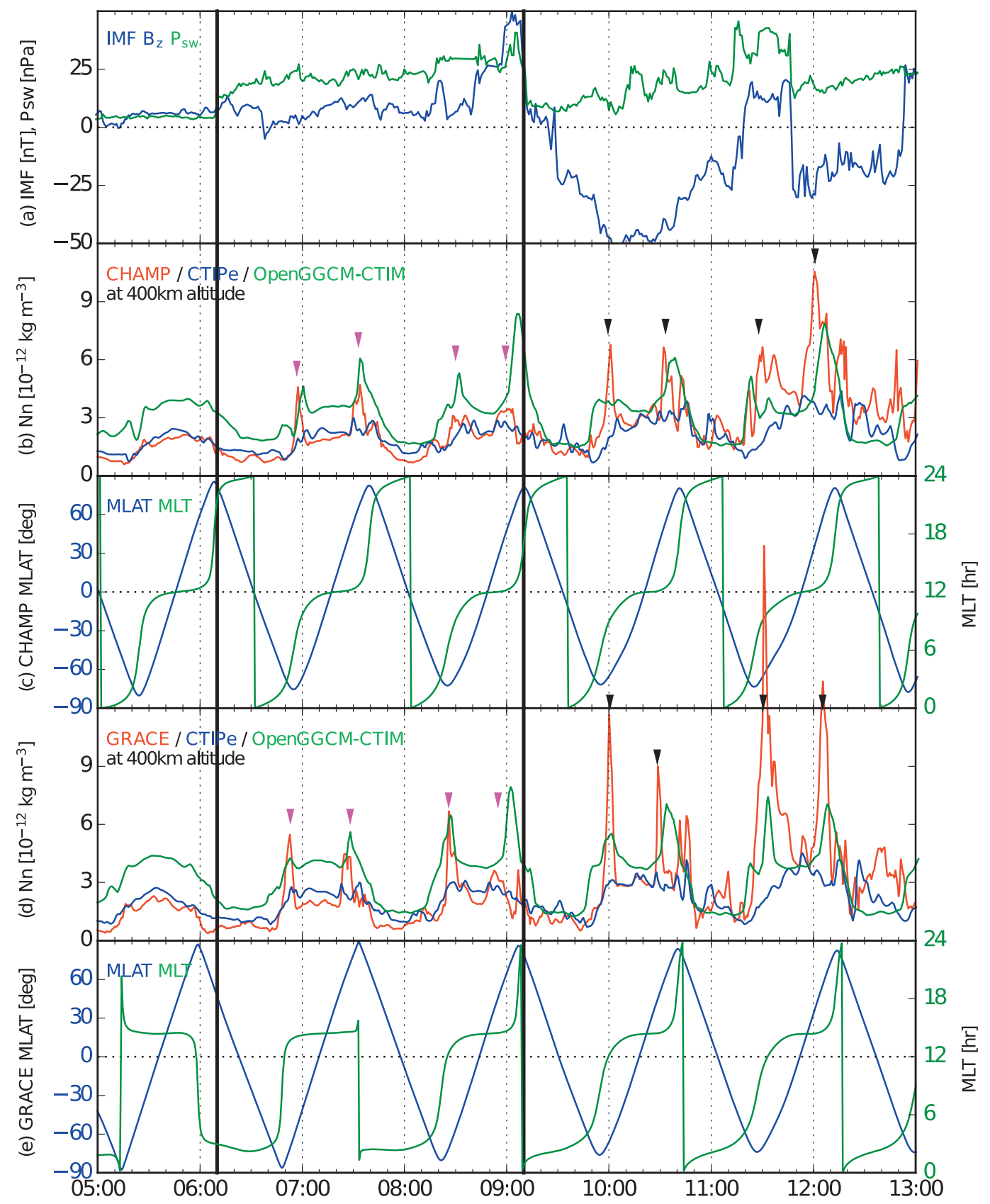

Fig. 2. Model-data comparison during 05:00-13:00 UT on August 24, 2005. (a) IMF (blue) and solar wind dynamic pressure (green), (b) CHAMP thermospheric mass densities (red) compared with the model results from CTIPe (blue) and OpenGGCM-CTIM (green), (c) CHAMP magnetic latitude (blue) and magnetic local time (green), (d) GRACE thermospheric mass densities (red) compared with the model results from CTIPe (blue) and OpenGGCM-CTIM (green), and (e) GRACE magnetic latitude (blue) and magnetic local time (green). The magenta and black markers point to the density peaks during the strong $P_{\text {sw }}$ period and the storm time, respectively.

energy input calculated from OpenGGCM with current SW drivers. Although the thermospheric densities are higher than the observed values during the quiet period before the storm, their general increase/decrease patterns match well with the satellite observation especially before 13:00 UT. The coupled model also captures the localized thermospheric heating at high latitudes relatively well during the compression and during the early stage of the storm. Figure 2 shows this pattern in more detail and clarity.

OpenGGCM-CTIM shows some discrepancies with data. Note that the OpenGGCM-CTIM does not include the mid- and low-latitude ionosphere-plasmasphere physics, which contributes to the new global IT circulation at the later phase of geomagnetic storms. It is not surprising, therefore, that the OpenGGCM-CTIM could not capture the density peaks observed at the mid- and low-latitude thermosphere (for example, see the peak at $\sim 13: 20$ UT in Fig. 1). The coupled model also produces higher thermospheric densities than the observations during the quiet time. Usually, the IT models need to run several days before a target event in order to stabilize the ionosphere and thermosphere, whose time scales for a steady state are longer than in the magnetosphere. However, the coupled 

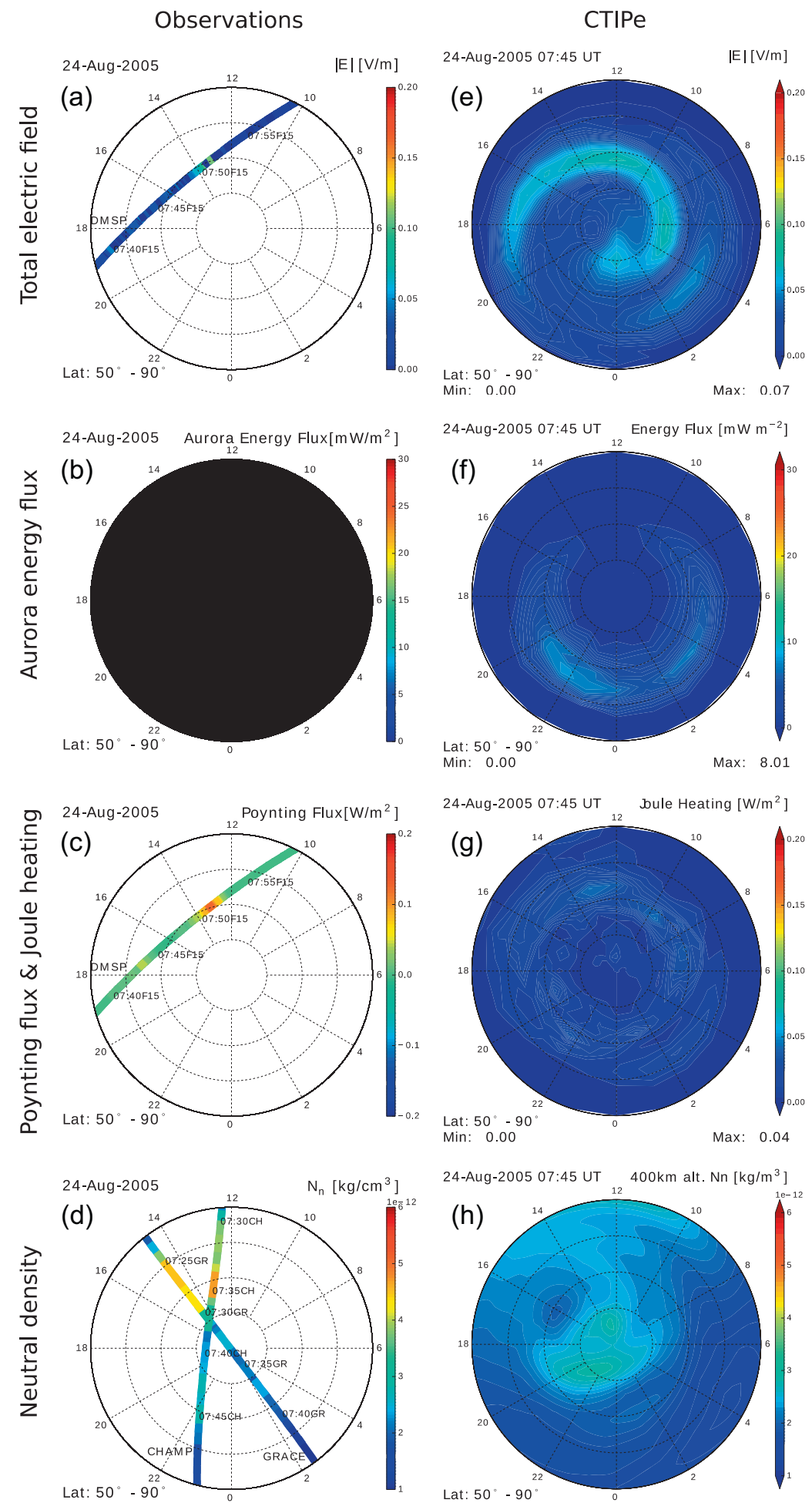

24-Aug-2005 07:45 UT $\quad 400 \mathrm{~km}$ alt. Nn $\left[\mathrm{kg} / \mathrm{m}^{3}\right]$
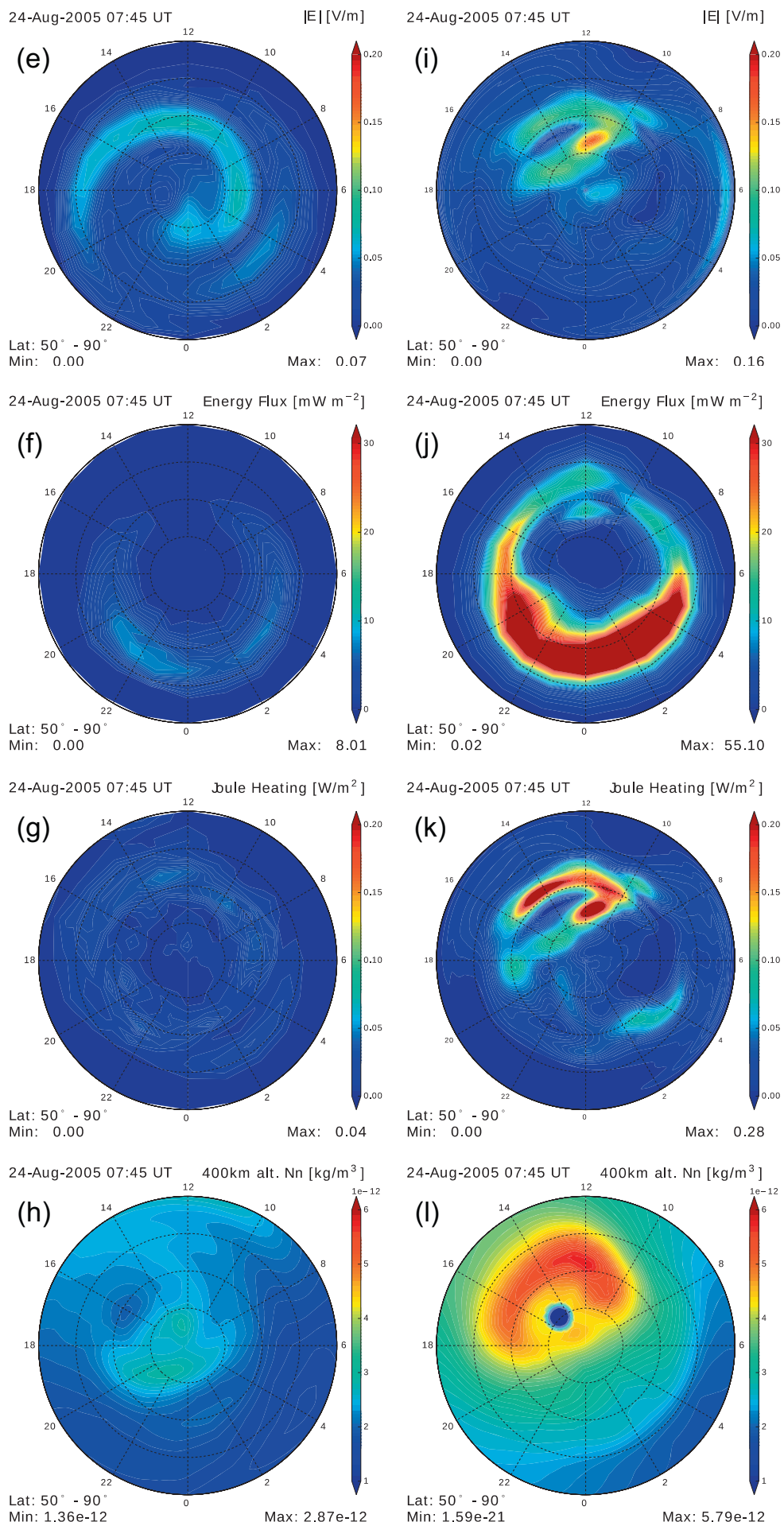

24-Aug-2005 07:45 UT Energy Flux $\left[\mathrm{mW} \mathrm{m}^{-2}\right]$
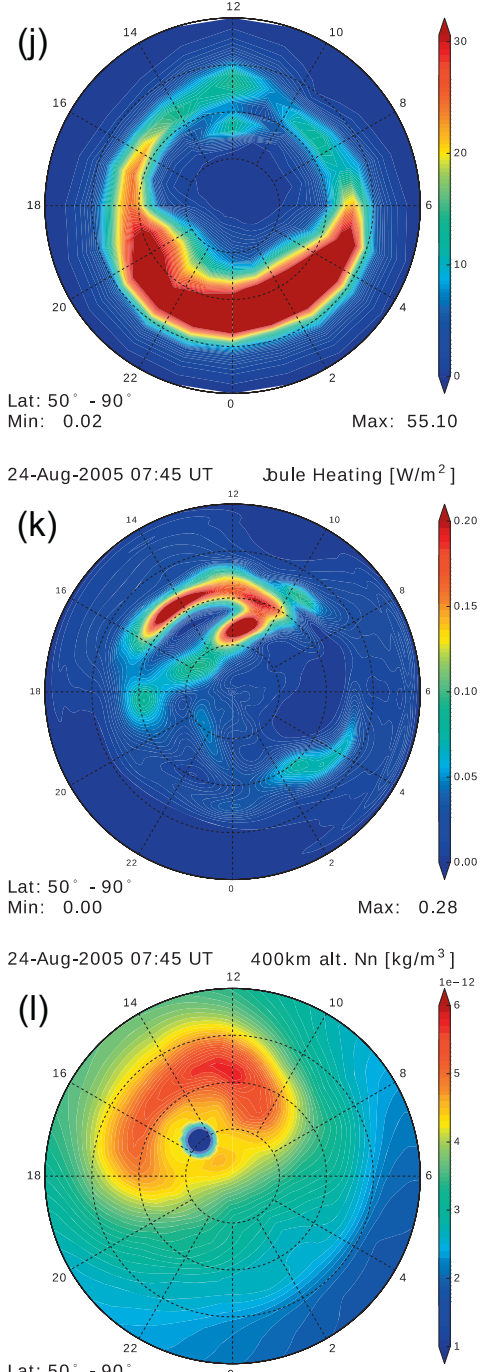

Lat: $50^{\circ}-90^{\circ}$

Max: $5.79 \mathrm{e}-12$

Fig. 3. The satellite observations (left), CTIPe model inputs and results (middle), and OpenGGCM-CTIM model inputs and results (right) projected on the northern hemisphere. (a) DMSP F15 total electric field, (b) DMSP F15 auroral energy flux, (c) DMSP F15 Poynting Flux, (d) CHAMP and GRACE neutral densities at $400 \mathrm{~km}$ altitude, (e) CTIPe total electric field input, (f) CTIPe auroral energy flux input, (g) CTIPe height-integrated Joule heating, (h) CTIPe neutral density at $400 \mathrm{~km}$ altitude, (i) OpenGGCM-CTIM total electric field input, (j) OpenGGCM-CTIM auroral energy flux input, (k) OpenGGCM-CTIM height-integrated Joule heating, and (1) OpenGGCM-CTIM neutral density at $400 \mathrm{~km}$ altitude.

OpenGGCM-CTIM model used here started at 12:00 UT on August 23, 2005 to avoid costly computation of the MHD model. A half-day of stabilization period was not enough to reach the observed thermospheric densities, resulting in the coupled model densities having an offset from the observations and CTIPe densities. Despite this offset, the relative changes in the OpenGGCM-CTIM densities after the shock and the storm onset are remarkably similar to the relative changes seen in 
observations and in the same locations, namely at the dayside high latitudes. This is the result of the more current, dynamic, and updated magnetospheric energy input to the IT system.

Figure 3 shows another model-data comparison by projecting the satellite observations and the corresponding model parameters on a polar view of the northern hemisphere. The left column of Figure 3 displays near simultaneous orbit tracks by DMSP F15 (panels a, b, and c), and CHAMP and GRACE (panel d), shortly after the shock impact of $\sim 6: 10$ UT and after the magnetosphere was strongly compressed, which is typically followed by strong enhancements of auroral precipitation and ionospheric electric fields (Zesta et al. 2000; Boudouridis et al. 2003, 2008, 2011). Figures 3a-3c present the total electric field, auroral energy flux, and Poynting flux measured along the orbit track of DMSP F15, while Figure $3 \mathrm{~d}$ presents the thermospheric mass density observed along the tracks of CHAMP and GRACE. The times and abbreviated spacecraft names next to each trajectory indicate where and when each spacecraft was located. The right two columns of Figure 3 display the model inputs and results from CTIPe and OpenGGCM-CTIM. Figures $3 \mathrm{e}-3 \mathrm{~h}$ show total electric field, auroral energy flux, height-integrated Joule heating, and neutral density obtained at 7:45 UT from CTIPe, and Figures 3i-31 show those same parameters from OpenGGCM-CTIM.

The DMSP F15 spacecraft observes $\sim 120 \mathrm{mV} / \mathrm{m}$ of enhanced total electric field near 14 MLT and $70^{\circ}$ MLAT as seen in Figure 3a. While the CTIPe input in Figure 3e shows smaller electric field strength around $\sim 80 \mathrm{mV} / \mathrm{m}$ at the same location, the OpenGGCM-CTIM input in Figure 3i shows $\sim 120 \mathrm{mV} / \mathrm{m}$ of total electric field that is comparable to the DMSP data. The DMSP F15 also observes $\sim 25 \mathrm{~mW} / \mathrm{m}^{2}$ of strong auroral energy flux near 17 MLT and $65^{\circ}$ MLAT as well as $\sim 8 \mathrm{~mW} / \mathrm{m}^{2}$ of moderate auroral energy flux near $12.5 \mathrm{MLT}$ and $68^{\circ}$ MLAT (see Fig. 3b). The CTIPe aurora input in Figure $3 \mathrm{f}$ significantly underestimates the observed auroral energy flux by providing $\sim 6 \mathrm{~mW} / \mathrm{m}^{2}$ and $\sim 0 \mathrm{~mW} / \mathrm{m}^{2}$ at the two auroral precipitation regions of DMSP. On the other hand, the OpenGGCM-CTIM provides $\sim 22 \mathrm{~mW} / \mathrm{m}^{2}$ and $\sim 12 \mathrm{~mW} /$ $\mathrm{m}^{2}$ of auroral energy flux at the two aurora regions as seen in Figure 3j, showing good agreement with the DMSP data. The top two rows of Figure 3 indicate that the OpenGGCMCTIM input reproduces the real magnetospheric energy input better than the empirical input used by CTIPe.

The DMSP Poynting flux observations in Figure $3 \mathrm{c}$ show strong electromagnetic energy deposition around 7:50 UT near 12-14 MLAT and $\sim 70^{\circ}$ MLAT. Because the Poynting flux energy is deposited into the IT system mainly via Joule heating (Fedrizzi et al. 2012), the high Poynting flux regions generally match with the strong Joule heating regions. The OpenGGCMCTIM predicts enhanced Joule heating where DMSP observes strong Poynting flux. In fact, the simulation shows a wider dayside region where Joule heating is enhanced, but the spatial dynamics cannot be captured by the single DMSP satellite. On the other hand, CTIPe predicts very weak Joule heating due to its underestimation of the total electric field and aurora precipitation input. Figure 3d shows that both CHAMP and GRACE observe thermospheric density enhancements around 7:30 UT near $12-16$ MLT and $55^{\circ}-75^{\circ}$ MLAT as the result of the strong DMSP Poynting flux. While the CTIPe results in Figure $3 \mathrm{~h}$ miss the density increase, the OpenGGCM-CTIM results in Figure 31 catch the enhanced neutral densities near the highlatitude dayside region where CHAMP and GRACE observe the strong neutral densities.
It is important to note, that the comparison is only qualitative due to the limitations of the OpenGGCM-CTIM coupled model. These result from the fact that the MHD theory approximates the magnetospheric behavior, not including all the physics that governs the near-Earth space environments. OpenGGCM-CTIM calculates ionospheric electric fields from a current continuity equation that assumes complete closure of field-aligned currents in the ionosphere. It also calculates auroral precipitation using a few analytical aurora equations that simplify the complex auroral particle dynamics (Kennel \& Petschek 1966; Knight 1973; Lyons et al. 1979). In spite of the limitations, the OpenGGCM-CTIM input shows better agreement with the DMSP observations in both magnitude and location than CTIPe input (see Fig. 3). This leads to better reproduction of the thermospheric density enhancements observed in the high-latitude dayside region during the $P_{\mathrm{sw}}$ impact and the early phase of a geomagnetic storm. On the other hand, CTIPe shows better agreement with the quiet-time thermospheric density than OpenGGCM-CTIM. CTIPe misses the localized high-latitude density peaks during 6:1012:10 UT in spite the fact that CTIPe is overall superior to CTIM. The poor performance of CTIPe in the storm early phases can be explained by the absence of dynamic magnetospheric energy input.

To understand details of the input difference and its impact on the IT system, Figures $4 a-4 c$ (Figs. 5a-5c) display ionospheric potentials, auroral precipitation, and thermospheric density at $400 \mathrm{~km}$ altitude on the northern hemisphere, obtained from CTIPe (OpenGGCM-CTIM). The panels of each column are obtained during the quiet period (6:00 UT), after the $P_{\mathrm{sw}}$ impact (6:45 UT), and after the main storm onset (9:45 UT), respectively. Figure 4 a shows the Weimer model ionospheric potentials used as the CTIPe input for the three representative instances. Both positive and negative potentials intensify after the $P_{\mathrm{sw}}$ impact and the storm onset. The cross polar cap potential (CPCP), the difference between maximum and minimum potentials in the northern ionosphere, changes from $\sim 85 \mathrm{kV}$ at $6: 00 \mathrm{UT}$ to $\sim 141 \mathrm{kV}$ at $6: 45 \mathrm{UT}$ and then to $\sim 192 \mathrm{kV}$ at 9:45 UT. Figure 5a presents OpenGGCM-CTIM ionospheric potentials calculated from the widely used MI coupling Eq. (6). The coupled MIT model also produces intensification of electric potentials after the $P_{\mathrm{sw}}$ impact and the storm onset. The CPCP increases from $\sim 110 \mathrm{kV}$ at $6: 00 \mathrm{UT}$ to $\sim 172 \mathrm{kV}$ at $6: 45 \mathrm{UT}$ and then to $\sim 194 \mathrm{kV}$ at $9: 45 \mathrm{UT}$, but there are distinct differences in the potential patterns of the two models, particularly in the size and shape of the morning and afternoon convection cells. The OpenGGCM-CTIM patterns include the IMF $B_{y}$ effects that are not as evident in the statistical Weimer patterns.

The auroral precipitation patterns appear different in the two models. CTIPe uses a total of seven aurora maps obtained from TIROS-NOAA statistics and selects one of the seven maps every minute depending on the hemispheric power index. Figure $4 \mathrm{~b}$ shows precipitation patterns relevant to the three instances. Figure 5b shows OpenGGCM-CTIM aurora precipitation calculated from Eqs. (1)-(5) using the plasma quantities and field-aligned currents near the inner magnetosphere boundary.

The CTIPe input aurora precipitation strengthens mostly in the nightside region after the $P_{\mathrm{sw}}$ impact (middle panel of Fig. 4b). In contrast, the coupled model shows enhanced auroral precipitation not only in the nightside region but also in the dayside afternoon section near 12-15 MLT (middle panel of 

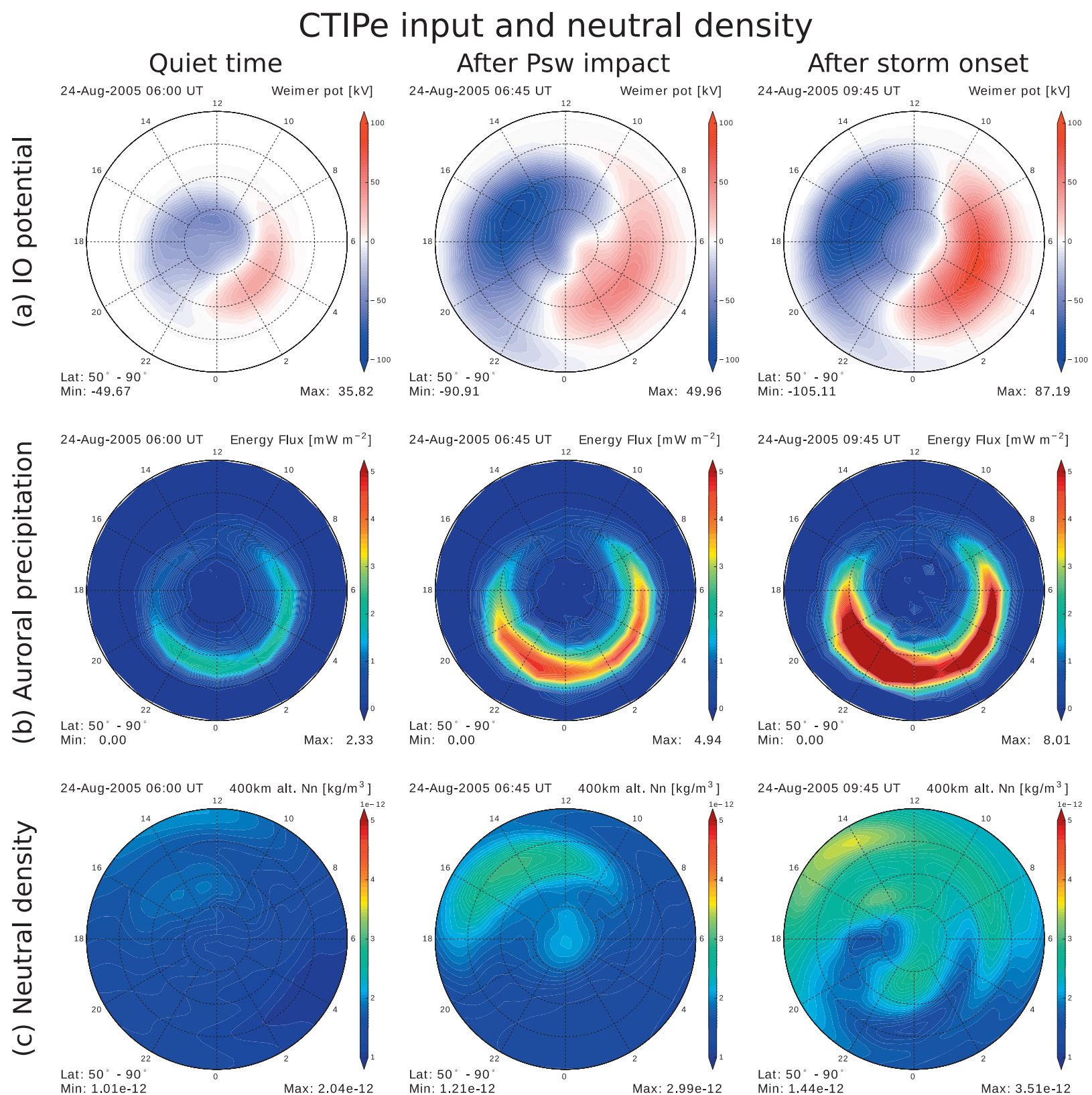

Fig. 4. CTIPe magnetospheric input and thermospheric neutral density during the quiet time, after the $P_{\mathrm{sw}}$ impact, and after the storm onset. (a) ionospheric electric potential, (b) auroral precipitation, and (c) thermospheric mass density in the $50^{\circ}-90^{\circ}$ MLAT.

Fig. 5b). That postnoon precipitation is typically the result of enhanced region 1 field-aligned currents after a compression, as reported in the previous studies of solar wind pressure shock impact (e.g., Zesta et al. 2000; Boudouridis et al. 2005; Shi et al. 2008). The coupled model also shows a much wider oval after the compression impact, particularly on the nightside, with the polar cap boundary having moved significantly poleward, as also reported in previous studies (Zesta et al. 2000; Boudouridis et al. 2004; Connor et al. 2014) as a result of enhanced nightside reconnection.

The CTIPe input aurora precipitation pattern during the storm main phase (right panel of Fig. 4b) shows a similar enhancement on the nightside but significantly stronger than the one after the $P_{\mathrm{sw}}$ impact. The hemispheric power index reaches its maximum value shortly after the storm onset and stays high (9 or 10) until 17:00 UT, except for a brief interval of HPI 8 during 14:33-14:43 UT. As a result, the aurora pattern seen in the right panel of Figure $4 \mathrm{~b}$ is repeatedly used as the CTIPe input during most of the storm period although more dynamic aurora is expected for a geomagnetic storm. In contrast, the storm-time aurora precipitation resulting from the OpenGGCM-CTIM simulation appears more dynamic (right panel of Fig. 5b), with a strong enhancement in the post-dusk local time region, most likely the result of the enhanced partial ring current precipitation during the main phase.

Figure 6 shows the aurora patterns during four instances of the first $30 \mathrm{~min}$ of the geomagnetic storm (from 9:10 to 9:40 UT). The auroral precipitation intensifies first in the afternoon section at 9:10 UT and localized around local midnight. Later by 9:40 UT, the aurora spreads over a broader MLT region including the entire nightside region, the 6-8 MLT, 


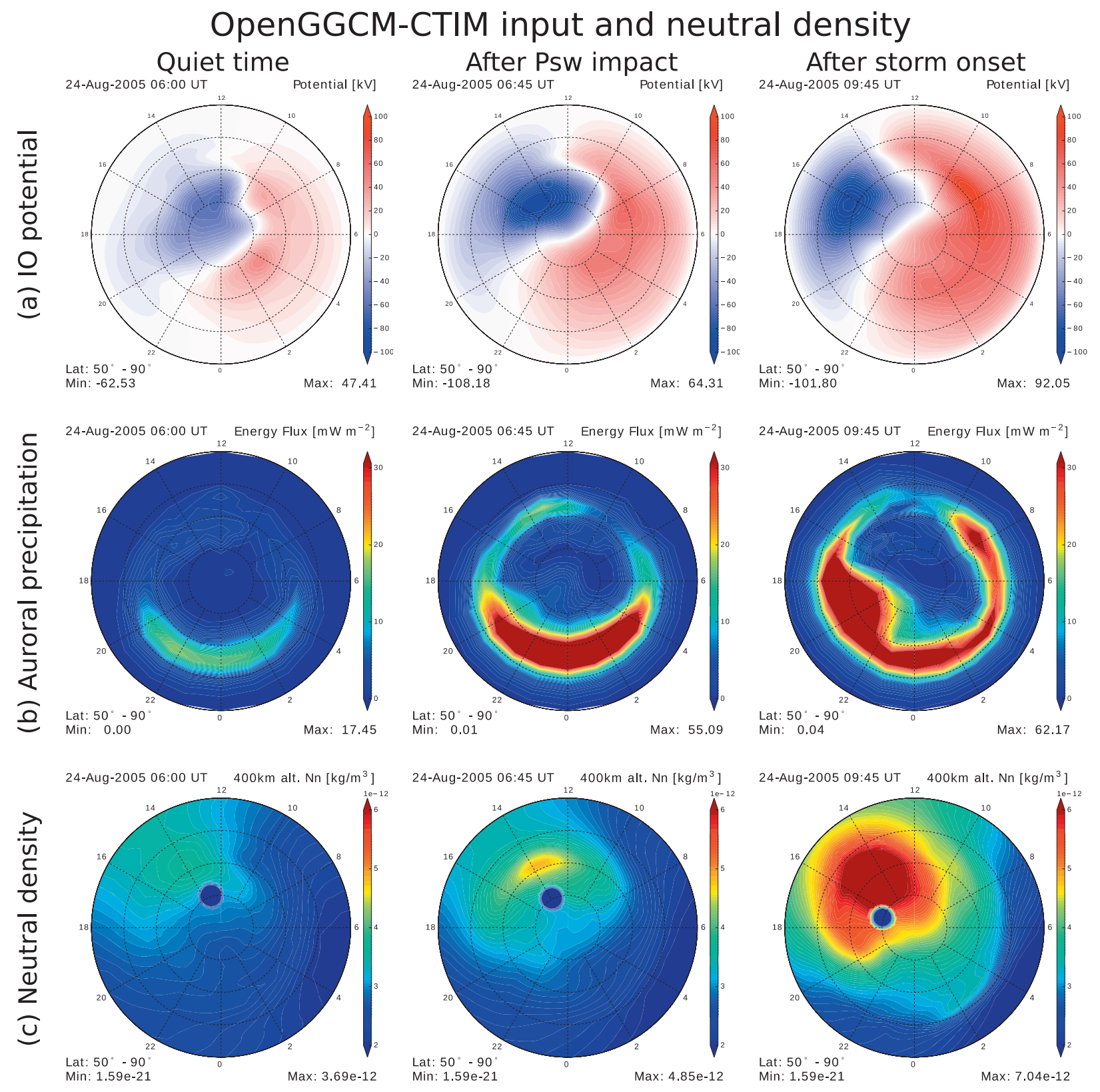

Fig. 5. OpenGGCM-CTIM magnetospheric input and thermospheric neutral density during the quiet time, after the $P_{\mathrm{sw}}$ impact, and after the storm onset. (a) Ionospheric electric potential, (b) auroral precipitation, and (c) thermospheric mass density in the $50^{\circ}-90^{\circ}$ MLAT.

and the 13-18 MLT. Note that in Figure 6 the OpenGGCMCTIM produces auroral precipitation consistently in the 13-16 MLT section although its energy flux is a bit weaker at 9:30 UT. This dayside precipitation is not present in the aurora input used by the CTIPe and that clearly affects the accuracy of the model results during storm time, particularly in the early phases of the storm. The importance of dayside aurora precipitation as the cause of localized high-latitude neutral density peaks has been reported in previous studies (Deng et al. 2013; Zhang et al. 2012, 2015b) and our work strengthens those conclusions.

Figures $4 \mathrm{c}$ and $5 \mathrm{c}$ show the neutral densities calculated from CTIPe and OpenGGCM-CTIM, respectively. The dark circle near the magnetic pole in Figure $5 \mathrm{c}$ indicates a geographic pole where the CTIM conducts no calculation and thus gives a default density. After the $P_{\mathrm{sw}}$ impact, both CTIPe and
OpenGGCM-CTIM observe neutral density enhancement in the afternoon section. However, the OpenGGCM-CTIM calculates a more significant density increase in the 12-15 MLT region where the strong auroral precipitation appears in Figure 5b. During the storm time, the neutral density increase is even bigger in the coupled MIT model. The maximum neutral density in OpenGGCM-CTIM jumps from $4.85 \times 10^{-12}$ at $6: 45$ UT to $7.04 \times 10^{-12} \mathrm{~kg} / \mathrm{m}^{3}$ at 9:45 UT, about four times larger than the density increase of CTIPe from $2.99 \times 10^{-12}$ to $3.51 \times 10^{-12} \mathrm{~kg} / \mathrm{m}^{3}$.

To understand the local neutral density peaks produced by OpenGGCM-CTIM, we display the height-integrated Pederson conductance, total electric field, and Joule heating of the northern ionosphere in Figures $7 \mathrm{a}-7 \mathrm{c}$, respectively, for the same three instances as in Figures 4 and 5, namely, quiet time, after $P_{\text {sw }}$ impact, and after the storm onset. After the $P_{\mathrm{sw}}$ impact, the 


\section{Auroral precipitation during the early phase of the 2005-08-24 storm}
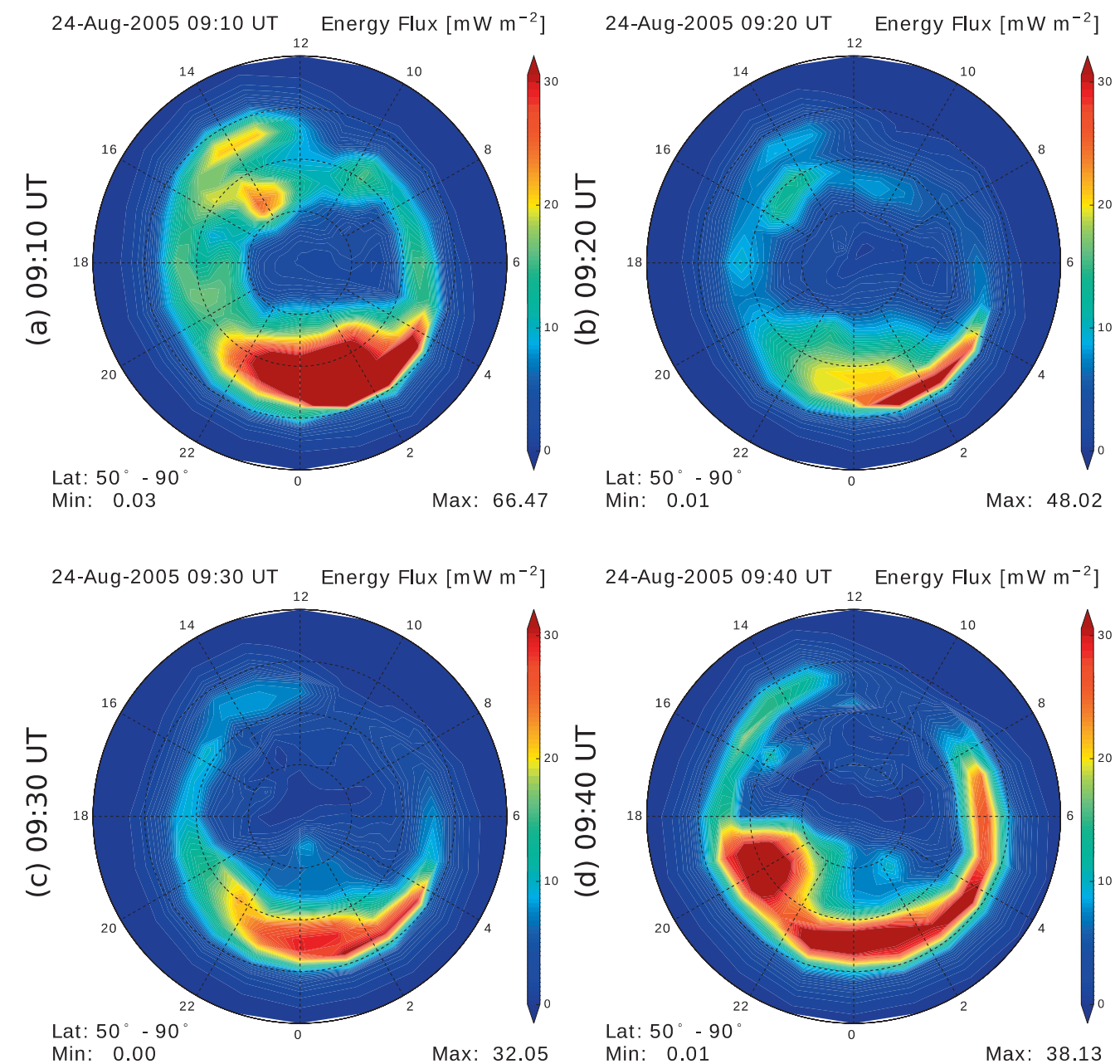

Fig. 6. OpenGGCM-CTIM aurora precipitation during the early phase of the August 24, 2005 geomagnetic storm (9:10-9:40 UT).

auroral precipitation increases in the 12-15 MLT and 65-70 ${ }^{\circ}$ MLAT region, ionizing the upper atmosphere and increasing Pederson conductance in this region. At the same time, the ionospheric electric fields increase near 8-16 MLT and at $\sim 70^{\circ}$ MLAT. The Joule heating is calculated from the equation:

$$
Q=\left(\boldsymbol{J}_{\mathbf{P}}+\boldsymbol{J}_{\mathbf{H}}\right) \cdot \boldsymbol{E}=\boldsymbol{J}_{\mathbf{P}} \cdot \boldsymbol{E}=\Sigma_{\mathrm{P}}|\boldsymbol{E}|^{2},
$$

where $Q$ is Joule heating, $\boldsymbol{J}_{\mathbf{P}}\left(\boldsymbol{J}_{\mathbf{H}}\right)$ is the Pederson (Hall) current density, $\boldsymbol{E}$ is the ionospheric electric field, and $\Sigma_{\mathrm{P}}$ is the height-integrated Pederson conductance. The increase of Pederson conductance and total electric field creates strong Joule heating near the 10-14 MLT section with the maximum strength near 13 MLT. These model results show that during the strong $P_{\mathrm{sw}}$ period, both electric field and Pederson conductance intensify near the high-latitude dayside ionosphere, warming the nearby thermosphere via Joule heating. The subsequent thermospheric upwelling increases neutral density at $400 \mathrm{~km}$ altitude.

During the first $30 \mathrm{~min}$ of the geomagnetic storm, the electric field strength is comparable to that after the $P_{\mathrm{sw}}$ impact although shown to be a bit weaker in the right panel of Figure $7 \mathrm{~b}$ for that particular instance. On the other hand, much stronger Pederson conductance appears near the 12-16 MLT during 9:10-9:45 UT due to the consistent auroral precipitation in this region. The stronger Joule heating appears in the afternoon section and persists for a long period after the storm onset. This warms up a broader region of the highlatitude thermosphere, causing the more pronounced neutral density increase in the entire afternoon section (see the right panel of Fig. 5c).

One may think that $30-65 \mathrm{~S}$ of Pederson conductance in the auroral oval (see Fig. 7a) is too high, but these values are in the range of previously measured conductance. For example, Semeter \& Doe (2002) derived Pederson conductance from the Sondrestrom incoherent scatter radar that ranges from 10 to $70 \mathrm{~S}$ depending on which aurora region the radar has pointed. Their study also mentioned that undersampling of auroral precipitation can cause the misrepresentation of true Pederson conductance by $100 \%$. Note that the CTIPe aurora input is much smaller than the DMSP observations (see Figs. $3 \mathrm{~b}$ and 3f). This indicates the limitation of the NOAA/TIROS statistical aurora maps especially for a storm study, due to their underestimation of the real auroral power input.

Due to the large Pederson conductance, OpenGGCM creates strong Joule heating in the IT system. Total Joule heating over the northern hemisphere in our coupled model starts from $\sim 250 \mathrm{GW}$ during a quiet time and then goes up to $2500 \mathrm{GW}$ during a geomagnetic storm. On the other hand, CTIPe produces less total Joule heating ranging from $\sim 150$ 

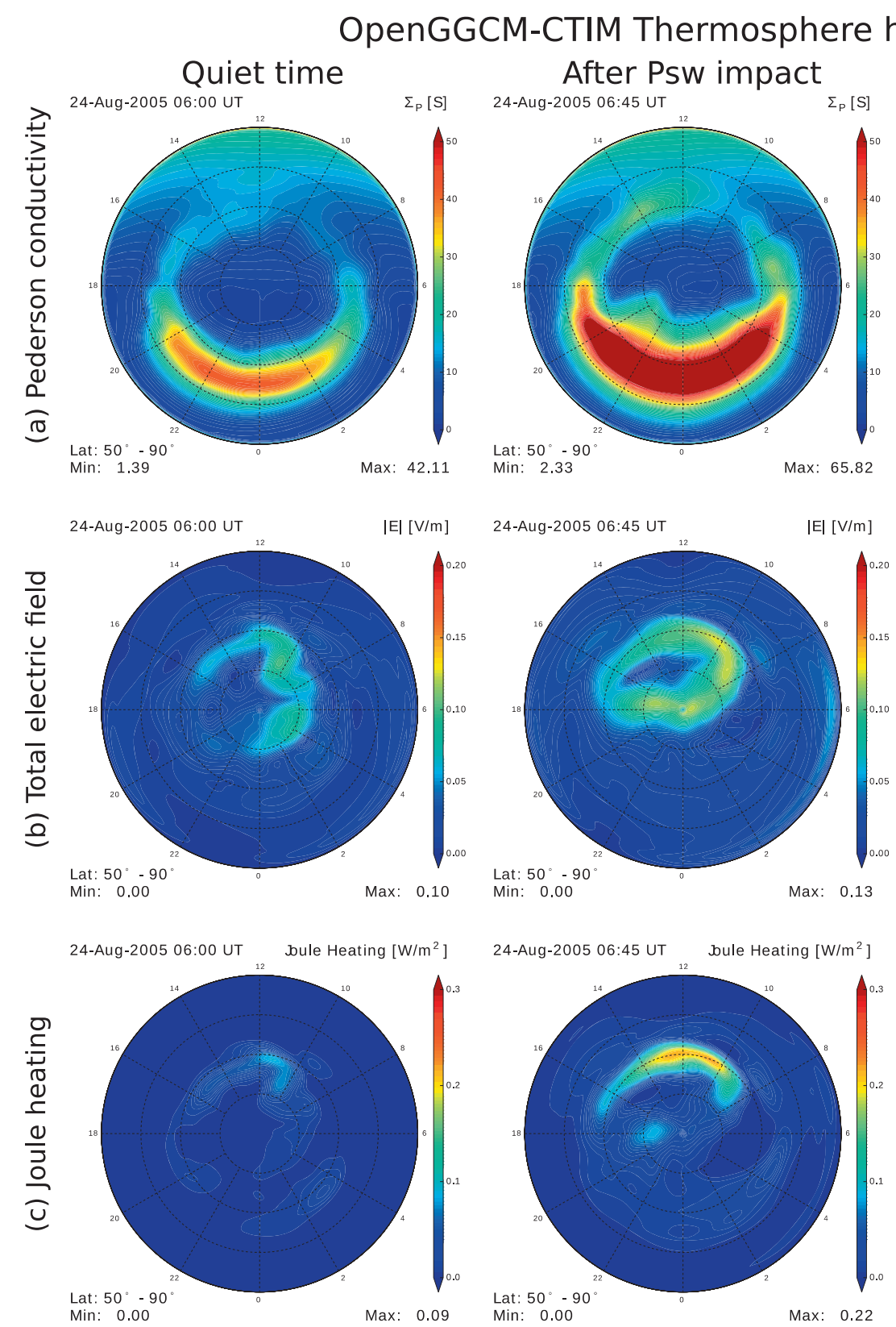

\section{heating}
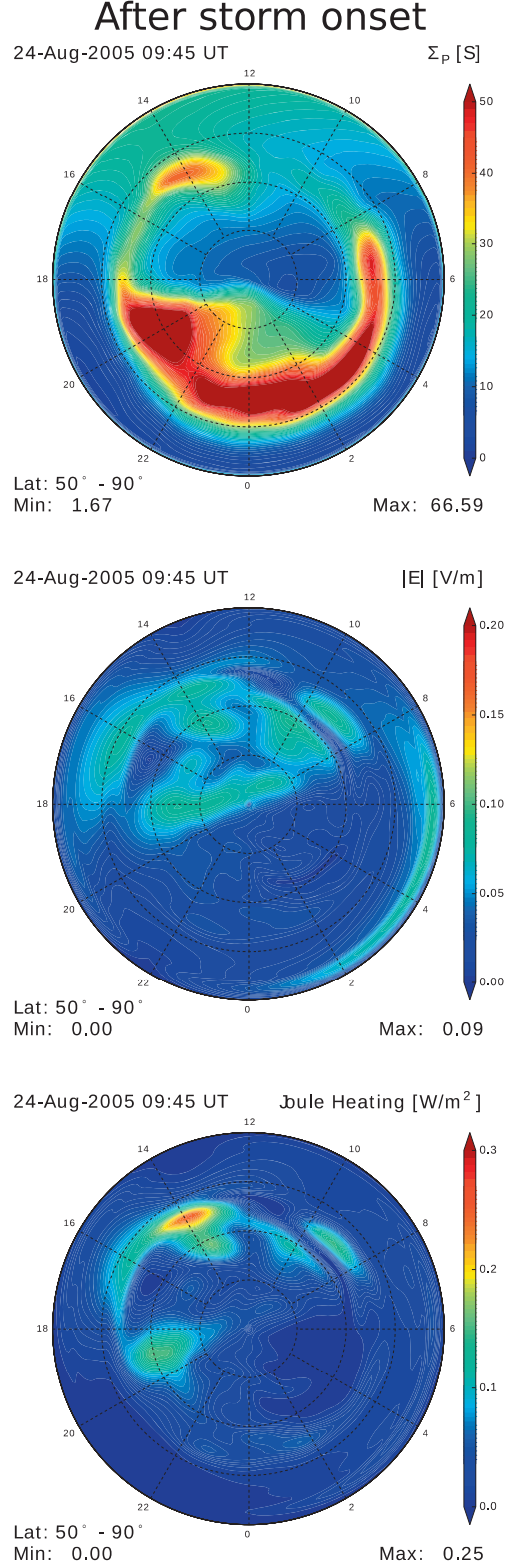

Fig. 7. OpenGGCM-CTIM results during the quiet time, after the $P_{\mathrm{sw}}$ impact, and after the storm onset. (a) Height-integrated Pederson conductance, (b) total electric field, and (c) Joule heating in the $50^{\circ}-90^{\circ}$ MLAT.

to $1500 \mathrm{GW}$ due to its reduced magnetospheric energy input. The total Joule heating of OpenGGCM-CTIM is high but comparable to the previously documented values. For example, Wilson et al. (2006) showed up to $\sim 4200 \mathrm{GW}$ of global Joule heating (i.e., $\sim 2100 \mathrm{GW}$ per each hemisphere) during geomagnetic storms.

Although model results of Pederson conductance and total Joule heating are comparable to the previously documented values, it is likely that OpenGGCM-CTIM overestimates auroral precipitation, particularly on the nightside, thus producing high Pederson conductance and Joule heating there. Our model produces over $30 \mathrm{~mW} / \mathrm{m}^{2}$ of auroral energy flux in the nightside auroral oval (see Figs. 3j, 5b, and 6). These fluxes are in the previously measured ranges (Galand et al. 2002; Hecht et al. 2008; Kaeppler et al. 2015) but much higher than what would be expected at quiet times from the observational evidence. The strong auroral precipitation in the model may be due to diffuse aurora that typically comprises more than $60 \%$ of the total auroral power (Newell et al. 2009). OpenGGCM-CTIM assumes a complete pitch-angle scattering of plasmasheet electrons on the nightside, although particle loss does not happen in all pitch angles. Our model introduces a uniform fudge factor, $\alpha$ in Eq. (2), over the whole hemisphere in order to adjust energy flux of diffuse aurora. The simulation for this study sets $\alpha$ at a default value 1 . Zhang et al. (2015a) showed that their new auroral precipitation model produces comparable results with OVATION Prime auroral model (Newell et al. 2014) when introducing spatial variation of this auroral fudge factor that ranges from 0 to 0.5 . By adopting a similar approach, OpenGGCM-CTIM can reduce the nightside auroral flux while maintaining the dayside aurora flux that agrees well with the DMSP observations (see Figs. $3 \mathrm{~b}$ and $3 \mathrm{j}$ ). 
The pertinent question to explore then is how the change of nightside auroral flux influences our MIT modeling results. The reduced nightside auroral flux can decrease conductances which in turn modify electric fields and Joule heating in the nightside region. Based on the current continuity Eq. (6), decreased conductances may increase electric fields if fieldaligned currents from the magnetosphere stay constant. Joule heating can increase if the electric field enhancement is strong enough to overcome the conductance decrease. However, the field-aligned currents do not stay constant. The electric field changes resulting from weaker conductances can affect the magnetospheric phenomena, altering the current patterns. Ridley et al. (2004) conducted multiple MHD simulations with the same solar wind/IMF conditions but with different Pederson conductances, showing that field-aligned currents decrease as the Pederson conductances decrease. If we expect similar behaviors in our MHD model (although our case is more complicated than his ideal cases), the reduced conductances can decrease field-aligned currents, thus resulting in less electric field changes due to the decrease of the two primary parameters in the current continuity equation. The big decrease in conductances and the minimal change in electric fields can lead to weaker Joule heating.

Due to the non-linear behavior of the MIT system, the impact of reduced nightside auroral flux can either increase or decrease electric fields and Joule heating in the nightside region. Such a modification would not critically harm the results of this paper that focuses on the dayside neutral density phenomena. Based on the comparison between the DMSP and modeled auroral fluxes, the auroral fudge factor would be close to 1 so little change is expected in the dayside region. Our focus here is only at the early hours after the shock and storm onset, and we see sharp enhancements at polar latitudes and first on the dayside cusp region. However, the state and structure of the nightside IT system can also affect the MIT coupling at geomagnetically active times (e.g., storms, steady magnetospheric convection intervals, substorms, sawteeth events, and sudden compressions). Under such times large amounts of energy are deposited in the aurora on the nightside and dynamically affect the state of the ionosphere and thermosphere over all local times. Future MIT modeling needs to consider all such effects.

The OpenGGCM-CTIM model simulates the stormtime IT system using an older version of the ionospherethermosphere model that does not include the upgrades of the CTIPe. While the coupling of the CTIPe with the OpenGGCM is our future project, the presented OpenGGCM-CTIM simulations capture the high-latitude responses after the $P_{\mathrm{sw}}$ impact and for the few hours after the storm onset with much higher physical accuracy. Our results demonstrate that in order to understand the physics behind the dynamic response of the ionosphere and thermosphere to storm-time phenomena, the auroral precipitation and electromagnetic energy inputs are critical. A coupled magnetosphere-IT model must be constructed to carry dynamic inputs into the IT model and the realistic IT feedback must be entered back into the global magnetospheric model continuously.

\section{Summary and concluding remarks}

We emphasize necessity of the physics-based magnetospheric energy input in order to advance predictability of the ionosphere-thermosphere system during strong geomagnetic activity. As a realistic magnetospheric input provider, we investigate the performance of the OpenGGCM global magnetosphere-ionosphere MHD model that is coupled with the CTIM global thermosphere and high-latitude ionosphere model. The MIT coupling of OpenGGCM-CTIM starts from the OpenGGCM side by providing a physics-based magnetospheric input to CTIM. OpenGGCM calculates the ionospheric electric fields by assuming a field-aligned current closure in the ionosphere, and the auroral precipitation by inputting the MHD plasma quantities to simple analytical equations of diffuse and discrete aurora. Then, CTIM solves the ionospherethermosphere system self-consistently, providing the heightintegrated ionospheric conductance to OpenGGCM. Finally, OpenGGCM updates an electric field pattern using the CTIM conductance and the MHD field-aligned currents.

We simulated a geomagnetic storm on August 24, 2005 using the OpenGGCM-CTIM and the CTIPe, and compared the two model results with observations of DMSP, CHAMP, and GRACE spacecraft. Since the CTIPe model includes more IT physics such as a low-latitude ionosphere and a plasmasphere, it is expected to provide better IT prediction than CTIM. However, CTIPe misses the high-latitude thermospheric density enhancement during strong $P_{\mathrm{sw}}$ period and the early stage of the storm, while the coupled MIT model catches the density peaks reasonably well. Unlike the CTIPe input models, the OpenGGCM-CTIM more faithfully produces localized increase of auroral precipitation and ionospheric electric fields in the high-latitude dayside region, causing strong Joule heating, thermospheric upwelling, and neutral density enhancement at $400 \mathrm{~km}$ altitude. Our results demonstrate that a physics-based magnetospheric energy input is critical to improve the storm-time IT prediction.

Although this paper provides promising results of an MIT coupling model, both magnetosphere MHD models and IT models have limitations that need to be addressed in future. First of all, OpenGGCM and other single fluid MHD models cannot fully address ring current physics, thus producing weak region 2 field-aligned currents. Impact of the region 2 currents on the MIT coupling system is likely underestimated in the MHD results, and the diffuse aurora patterns are almost certainly affected. This problem can be alleviated by coupling the MHD models with ring current models (Rice Convection Model and Comprehensive Ring Current Model for example). Additionally, MHD models cannot simulate auroral particle dynamics due to their fluid approach. The MHD modelers take different approaches to parameterize auroral precipitation (Raeder 2003; Ridley et al. 2004; Zhang et al. 2015a). However, these approaches are empirically or analytically obtained with assumptions that simplify auroral process and most importantly the spatial distribution of precipitation patterns. Recent works have demonstrated how important meso-scale and small-scale structures are in the accurate determination of the Joule heating, but both are missed by empirical and current analytical approaches (Deng et al. 2009). Development of a physics-based self-consistent aurora particle model is necessary for future MIT coupling studies.

Current IT models such as CTIPe, TIEGCM, and GTIM (Global Ionosphere Thermosphere Model) may underestimate height-integrated conductances. They adopt empirical techniques like Roble \& Ridley (1987) and Fuller-Rowell \& Evans (1987) to calculate electron impact ionization rates that determine ionospheric conductances. However, these techniques assume Maxwellian energy distribution of precipitating electrons, producing errors when electron energy distribution is 
deviated from the Maxwellian. For instance, 37\% of precipitating electrons follow kappa distribution (McIntosh \& Anderson 2014). A power law tail of kappa distribution produces more flux at high energies, strengthening ionization at low-altitude atmosphere and increasing Hall conductance. Additionally, the current ionization models cover electron energy from several hundreds of $\mathrm{eV}$ to several tens of $\mathrm{keV}$, thus ignoring the impact of soft electron precipitation and secondary electrons that can produce strong ionization at high-latitude atmosphere and high Pederson conductance (Thayer \& Semeter 2004; Huang et al. 2014; Khazanov et al. 2014). Since spatiotemporal distribution of ionospheric conductance influences electric field patterns and thus MI coupling processes, realistic input of energy distribution and accurate calculation of ionization rates are necessary to understand the MIT coupling dynamics. Recently, Fang et al. (2010) introduced a new ionization model applicable to any electron spectrum with wider energy coverage $(100 \mathrm{eV} \sim 1 \mathrm{MeV})$. Inclusion of Fang's ionization model can be a good starting point to improve the current IT models.

It is difficult to answer how the model limitations influence our understanding of a global MIT coupling system due to its non-linear nature. Changes in any one of MIT coupling parameters (field-aligned currents, auroral precipitation, or conductances) can vary ionospheric electric fields, modify the whole MIT system, and influence other MIT parameters. Combined impact of all parameter changes can be even more complex and is certainly not known due to limited observational coverage and the absence of appropriate physics in current modeling tools. These issues can be addressed only in the future as the MIT modeling tools include proper inner magnetosphere physics and auroral particle dynamics, and as global coverage of observations is achieved throughout the magnetosphere-ionosphere-thermosphere system.

Acknowledgements. The work by H. K. Connor was supported by the National Science Foundation under Award No. 1331368 and by an appointment to the NASA Postdoctoral Program at the Goddard Space Flight Center, administered by Oak Ridge Associated Universities through a contract with NASA. The work by E. Zesta and Y. Shi was supported by Grant 13-SRITM13_2-0011 from NASA Heliophysics Supporting Research program. The work by Dr. Raeder was supported by Grant FA95501210264 from the Air Force Office of Sponsored Research and by Grant AGS-1143895 from the National Science Foundation. Computations were performed on Trillian, a Cary XE6m-200 supercomputer at UNH supported by the NSF MRI program under Grant PHY-1229408. H. K. Connor thanks C. Y. Huang and Y.-J. Su for useful discussions. The editor thanks two anonymous referees for their assistance in evaluating this paper.

\section{References}

Boudouridis, A., E. Zesta, L.R. Lyons, P.C. Anderson, and D. Lummerzheim. Effect of solar wind pressure pulses on the size and strength of the auroral oval. J. Geophys. Res., 108 (A4), 8012, 2003, DOI: 10.1029/2002JA009373.

Boudouridis, A., E. Zesta, L.R. Lyons, P.C. Anderson, and D. Lummerzheim. Magnetospheric reconnection driven by solar wind pressure fronts. Ann. Geophys., 22, 1367-1378, 2004.

Boudouridis, A., E. Zesta, L.R. Lyons, P.C. Anderson, and D. Lummerzheim. Enhanced solar wind geoeffectiveness after a sudden increase in dynamic pressure during southward IMF orientation. J. Geophys. Res., 110, A05214, 2005, DOI: 10.1029/2004JA010704.
Boudouridis, A., E. Zesta, L.R. Lyons, P.C. Anderson, and A.J. Ridley. Temporal evolution of the transpolar potential after a sharp enhancement in solar wind dynamic pressure. Geophys. Res. Lett., 35, L02101, 2008,

DOI: 10.1029/2007GL031766.

Boudouridis, A., L.R. Lyons, E. Zesta, J.M. Weygand, A.J. Ribeiro, and J.M. Ruohoniemi. Statistical study of the effect of solar wind dynamic pressure fronts on the dayside and nightside ionospheric convection. J. Geophys. Res., 116, A10233, 2011, DOI: 10.1029/2011JA016582.

Chiu, T. An improved phenomenological model of ionospheric density. J. Atmos. Terr. Phys., 37, 1563-1570, 1975.

Codrescu, M.V., C. Negrea, M. Fedrizzi, T.J. Fuller-Rowell, A. Dobin, N. Jakowsky, H. Khalsa, T. Matsuo, and N. Maruyama. A real-time run of the Coupled Thermosphere Ionosphere Plasmasphere Electrodynamics (CTIPe) model. Space Weather, 10, S02001, 2012, DOI: 10.1029/2011SW000736.

Connor, H.K., E. Zesta, D.M. Ober, and J. Raeder. The relation between transpolar potential and reconnection rates during sudden enhancement of solar wind dynamic pressure: OpenGGCM-CTIM results. J. Geophys. Res. [Space Phys.], 119, 3411-3429, 2014, DOI: 10.1002/2013JA019728.

Crowley, G., D.J. Knipp, K.A. Drake, J. Lei, E. Sutton, and H. Lühr. Thermospheric density enhancements in the dayside cusp region during strong BY conditions. Geophys. Res. Lett., 37, L07110, 2010, DOI: 10.1029/2009GL042143.

Deng, Y., A. Maute, A.D. Richmond, and R.G. Roble. Impact of electric field variability on Joule heating and thermospheric temperature and density. Geophys. Res. Lett., 36, L08105, 2009, DOI: 10.1029/2008GL036916.

Deng, Y., T.J. Fuller-Rowell, A.J. Ridley, D. Knipp, and R.E. Lopez. Theoretical study: influence of different energy sources on the cusp neutral density enhancement. J. Geophys. Res. [Space Phys.], 118, 2340-2349, 2013, DOI: 10.1002/jgra.50197.

Fang, X., C.E. Randall, D. Lummerzheim, W. Wang, G. Lu, S.C. Solomon, and R.A. Frahm. Parameterization of monoenergetic electron impact ionization. Geophys. Res. Lett., 37, L22106, 2010, DOI: $10.1029 / 2010$ GL045406.

Fedrizzi, M., T.J. Fuller-Rowell, and M.V. Codrescu. Global Joule heating index derived from thermospheric density physics-based modeling and observations. Space Weather, 10, S03001, 2012, DOI: 10.1029/2011SW000724.

Fuller-Rowell, T.J., and D.S. Evans. Height-integrated Pedersen and Hall conductivity patterns inferred from the TIROS-NOAA satellite data. J. Geophys. Res., 92 (A7), 7606-7618, 1987, DOI: 10.1029/JA092iA07p07606.

Fuller-Rowell, T.J., D. Rees, S. Quegan, R.J. Moffett, M.V. Codrescu, and G.H. Millward. A coupled thermosphere-ionosphere model (CTIM). In: R.W. Schunk, Editor, Solar-Terrestrial Energy Program: Handbook of Ionospheric Models, Cent. for Atmos. and Space Sci., Utah State Univ., Logan, Utah, 217-238, 1996.

Fuller-Rowell, T., M. Codrescu, N. Maruyama, M. Fredrizzi, E. Araujo-Pradere, S. Sazykin, and G. Bust. Observed and modeled thermosphere and ionosphere response to superstorms. Radio Sci., 42, RS4S90, 2007, DOI: 10.1029/2005RS003392.

Galand, M., D. Lummerzheim, A.W. Stephan, B.C. Bush, and S. Chakrabarti. Electron and proton aurora observed spectroscopically in the far ultraviolet. J. Geophys. Res., 107 (A7), 2002, DOI: 10.1029/2001JA000235.

Gilson, M.L., J. Raeder, E. Donovan, Y.S. Ge, and L. Kepko. Global simulation of proton precipitation due to field line curvature during substorms. J. Geophys. Res., 117, A05216, 2012, DOI: 10.1029/2012JA017562.

Hecht, J.H., T. Mulligan, D.J. Strickland, A.J. Kochenash, Y. Murayama, et al. Satellite and ground-based observations of auroral energy deposition and the effects on thermospheric composition during large geomagnetic storms: 1. Great geomagnetic storm of 20 November. J. Geophys. Res., 113, A01310, 2008, DOI: 10.1029/2007JA012365. 
Heelis, R.A., J.K. Lowell, and R.W. Spiro. A model of the highlatitude ionospheric convection pattern. J. Geophys. Res., 87, 6339, 1982.

Huang, Y., C.Y. Huang, Y.-J. Su, Y. Deng, and X. Fang. Ionization due to electron and proton precipitation during the August 2011 storm. J. Geophys. Res. [Space Phys.], 119, 3106-3116, 2014, DOI: 10.1002/2013JA019671.

Kaeppler, S.R., D.L. Hampton, M.J. Nicolls, A. Strømme, S.C. Solomon, J.H. Hecht, and M.G. Conde. An investigation comparing ground-based techniques that quantify auroral electron flux and conductance. J. Geophys. Res. [Space Phys.], 120, 9038-9056, 2015, DOI: 10.1002/2015JA021396.

Kennel, C.F., and H.E. Petschek. Limit on stably trapped particle fluxes. J. Geophys. Res., 71, 1, 1966.

Kelley, M.C. The Earth's Ionosphere, Academic Press, New York, 1989

Khazanov, G.V., A. Glocer, and E.W. Himwich. Magnetosphereionosphere energy interchange in the electron diffuse aurora. J. Geophys. Res. [Space Phys.], 119, 171-184, 2014, DOI: 10.1002/2013JA019325.

Knight, S. Parallel electric fields. Planet. Space Sci., 21, 741, 1973.

Knipp, D.J., and B.A. Emery. Mapping ionospheric substorm response. Adv. Space Res., 20 (4/5), 895-905, 1997.

Knipp, D., S. Eriksson, L. Kilcommons, G. Crowley, J. Lei, M. Hairston, and K. Drake. Extreme Poynting flux in the dayside thermosphere: examples and statistics. Geophys. Res. Lett., 38, L16102, 2011, DOI: 10.1029/2011GL048302.

Lei, J., W. Wang, A.G. Burns, S.C. Solomon, A.D. Richmond, M. Wiltberger, L.P. Goncharenko, A. Coster, and B.W. Reinisch. Observations and simulations of the ionospheric and thermospheric response to the December 2006 geomagnetic storm: initial phase. J. Geophys. Res., 113, A01314, 2008, DOI: 10.1029/2007JA012807.

Li, W., D. Knipp, J. Lei, and J. Raeder. The relation between dayside local Poynting flux enhancement and cusp reconnection. J. Geophys. Res., 116, A08301, 2011, DOI: 10.1029/2011JA016566.

Lyons, L.R., D. Evans, and R. Lundin. An observed relation between magnetic field aligned electric fields and downward electron energy fluxes in the vicinity of auroral forms. J. Geophys. Res., 84, 457, 1979.

McIntosh, R.C., and P.C. Anderson. Maps of precipitating electron spectra characterized by Maxwellian and kappa distributions. J. Geophys. Res. [Space Phys.], 119, 10116-10132, 2014, DOI: $10.1002 / 2014 J A 020080$.

Millward, G.H., R.J. Moffett, S. Quegan, and T.J. Fuller-Rowell. A coupled thermosphere-ionosphere-plasmasphere model (CTIP). In: R.W. Schunk, Editor, Solar-Terrestrial Energy Program: Handbook of Ionospheric Models, Cent. for Atmos. and Space Sci., Utah State Univ., Logan, Utah, 239-279, 1996.

Millward, G.H., I.C.F. Müller-Wodarg, A.D. Aylward, T.J. FullerRowell, A.D. Richmond, and R.J. Moffett. An investigation into the influence of tidal forcing on $\mathrm{F}$ region equatorial vertical ion drift using a global ionosphere-thermosphere model with coupled electrodynamics. J. Geophys. Res., 106 (A11), 24733-24744, 2001, DOI: $10.1029 / 2000 J A 000342$.

Newell, P.T., T. Sotirelis, and S. Wing. Diffuse, monoenergetic, and broadband aurora: the global precipitation budget. J. Geophys. Res., 114, A09207, 2009, DOI: 10.1029/2009JA014326.

Newell, P.T., K. Liou, Y. Zhang, T. Sotirelis, L.J. Paxton, and E.J. Mitchell. OVATION Prime-2013: extension of auroral precipitation model to higher disturbance levels. Space Weather, 12, 368-379, 2014, DOI: 10.1002/2014SW001056.

Raeder, J. Global magnetohydrodynamics - a tutorial. In: J. Buechner, C.T. Dum, and M. Scholer, Editors, Space Plasma Simulation, Lecture Notes in Physics, Springer-Verlag, Heidelberg, Germany, 615, 2003.

Raeder, J., and G. Lu. Polar cap potential saturation during large geomagnetic storms. Adv. Space Res., 36, 1804, 2005.
Raeder, J., R.L. McPherron, L.A. Frank, S. Kokubun, G. Lu, et al. Global simulation of the geospace environment modeling substorm challenge event. J. Geophys. Res., 106, 381, 2001 a.

Raeder, J., Y. Wang, and T. Fuller-Rowell. Geomagnetic storm simulation with a coupled magnetosphere-ionospherethermosphere model. In: P. Song, H.J. Singer, and G. Siscoe, Editors, Space Weather: Progress and Challenges in Research and Applications, Geophys. Monogr. Ser., vol. 125, AGU, Washington, DC, 377-384, 2001b.

Raeder, J., D. Larson, W. Li, E.L. Kepko, and T. Fuller-Rowell. OpenGGCM simulations for the THEMIS mission. Space Sci. Rev., 141, 535-555, 2008, DOI: 10.1007/s11214-0421-5.

Richmond, A.D., and Y. Kamide. Mapping electrodynamic features of the high latitude ionosphere from localized observations. J. Geophys. Res., 93, 5741, 1988.

Richmond, A.D., and G. Lu. Upper-atmospheric effects of magnetic storms: a brief tutorial. J. Atmos. Sol. Terr. Phys., 62, 1115-1127, 2000, DOI: 10.1016/S1364-6826(00)00094-8.

Ridley, A.J., T.I. Gombosi, and D.L. DeZeeuw. Ionospheric control of the magnetosphere: conductance. Ann. Geophys., 22, 567-584, 2004, DOI: 10.5194/angeo-22-567-2004.

Robinson, R.M., R.R. Vondrak, K. Miller, T. Dabbs, and D. Hardy. On calculating ionospheric conductances from the flux and energy of precipitating electrons. J. Geophys. Res., 92, 2565, 1987.

Roble, R.G., and E.C. Ridley. An auroral model for the NCAR thermospheric general circulation model (TGCM). Ann. Geophys., 5, 369-382, 1987.

Schlegel, K., H. Lühr, J.P. St. Maurice, G. Crowley, and C. Hackert. Thermospheric density structures over the polar regions observed with CHAMP. Ann. Geophys., 23, 1659-1672, 2005.

Semeter, J., and R. Doe. On the proper interpretation of ionospheric conductance estimated through satellite photometry. J. Geophys. Res., 107, A8, 2002, DOI: 10.1029/2001JA009101.

Shi, Y., E. Zesta, and L.R. Lyons. Modeling magnetospheric current response to solar wind dynamic pressure enhancements during magnetic storms: 1 . Methodology and results of the 25 September 1998 peak main phase case. J. Geophys. Res., 113, A10218, 2008, DOI: 10.1029/2008JA013111.

Thayer, J.P., and J. Semeter. The convergence of magnetospheric energy flux in the polar atmosphere. J. Atmos. Sol. Terr. Phys., 66, 807-824, 2004.

Vasyliunas, V.M. Mathematical models of magnetospheric convection and its coupling to the ionosphere. In: B.M. McCormac, Editor, Particles and Fields in the Magnetosphere, D. Reidel, Norwell, Mass, 61-71, 1970.

Wang, W., M. Wiltberger, A.G. Burns, S. Solomon, T.L. Killeen, N. Maruyama, and J. Lyon. Initial results from the CISM coupled magnetosphere-ionosphere-thermosphere (CMIT) model: thermosphere ionosphere responses. J. Atmos. Sol. Terr. Phys., 66, 1425-1442, 2004, DOI: 10.1016/j.jastp.2004.04.008.

Wang, W., J. Lei, A.G. Burns, M. Wiltberger, A.D. Richmond, S.C. Solomon, T.L. Killeen, E.R. Talaat, and D.N. Anderson. Ionospheric electric field variations during a geomagnetic storm simulated by a coupled magnetosphere ionosphere thermosphere (CMIT) model. Geophys. Res. Lett., 35, L18105, 2008, DOI: 10.1029/2008GL035155.

Wang, W., J. Lei, A.G. Burns, S.C. Solomon, M. Wiltberger, J. Xu, Y. Zhang, L. Paxton, and A. Coster. Ionospheric response to the initial phase of geomagnetic storms: common features. J. Geophys. Res., 115, A07321, 2010, DOI: 10.1029/2009JA014461.

Weimer, D.R. Predicting surface geomagnetic variations using ionospheric electrodynamic models. J. Geophys. Res., 110, 12307, 2005, DOI: 10.1029/2005JA011270.

Wilson, G.R., D.R. Weimer, J.O. Wise, and F.A. Marcos. Response of the thermosphere to Joule heating and particle precipitation. J. Geophys. Res., 111, A10314, 2006, DOI: $10.1029 / 2005 J A 011274$. 
H.K. Connor et al.: Modeling of the coupled magnetosphere - ionosphere - thermosphere system

Zesta, E., H.J. Singer, D. Lummerzheim, C.T. Russell, L.R. Lyons, and M.J. Brittnacher. The effect of the January 10, 1997, pressure pulse on the magnetosphere-ionosphere current system. In: S. Ohtani, et al. Editors, Magnetospheric Current Systems, Geophys. Monogr. Ser., vol. 118, AGU, Washington, DC, 217-226, 2000.

Zhang, B., W. Lotko, O. Brambles, M. Wiltberger, W. Wang, P. Schmitt, and J. Lyon. Enhancement of thermospheric mass density by soft electron precipitation. Geophys. Res. Lett., 39, L20102, 2012, DOI: 10.1029/2012GL053519.
Zhang, B., W. Lotko, O. Brambles, M. Wiltberger, and J. Lyon. Electron precipitation models in global magnetosphere simulations. J. Geophys. Res. [Space Phys.], 120, 1-2, 2015a, DOI: $10.1002 / 2014 J A 020615$.

Zhang, B., R.H. Varney, W. Lotko, O.J. Brambles, W. Wang, J. Lei, M. Wiltberger, and J.G. Lyon. Pathways of F region thermospheric mass density enhancement via soft electron precipitation. J. Geophys. Res. [Space Phys.], 120, 5824-5831, 2015b, DOI: 10.1002/2015JA020999.

Cite this article as: Connor HK, Zesta E, Fedrizzi M, Shi Y, Raeder J, et al. Modeling the ionosphere-thermosphere response to a geomagnetic storm using physics-based magnetospheric energy input: OpenGGCM-CTIM results. J. Space Weather Space Clim., 6, A25, 2016, DOI: $10.1051 / \mathrm{swsc} / 2016019$. 